\title{
ELECTRON MICROSCOPIC ASSAY OF BACTERIAL BIOFILM FORMED ON INDWELLING URETHRAL CATHETERS
} By

\author{
MAGDA AZMY ${ }^{1}$, NADA NAWAR ${ }^{2}$, MONA MOHIEDDEN ${ }^{2}$, AND LILIAN WARILLE $^{3}$ \\ Department of Electron Microscopy, Theodor Bilharz Research Institute, Giza, Egypt ${ }^{1}$, \\ Department of Clinical and Chemical Pathology, Faculty of Medicine, Cairo University, \\ Egypt $^{2}$, Faculty of Medicine, Juba University, Sudan ${ }^{3}$
}

\begin{abstract}
Biofilm formation on indwelling urinary catheters is a leading cause of Urinary tract infection (UTI). Presence of biofilm is associated with increased bacterial resistance to antimicrobial therapy and resultant treatment failure. The study detected a reliable method for diagnosis of biofilm formation by comparing scanning electron microscopy (SEM) and tissue culture plate method (TCP). The work was conducted on 20 urinary catheters from patients ranging from 1.5 to 85 years with catheters that remained in situ for a period of 3 to 20 days. Samples of catheters for culture and SEM and samples of urine were taken at the same time. The correlation between renal conditions and biofilm formation was not significant $(\mathrm{p}=0.336)$. No significant correlation ( $\mathrm{p}=0.836,0.163$ respectively) was found between predisposing conditions (DM, renal insufficiency, diarrhea and impaired immunity) and development of Catheter associated urinary tract infection ( CAUTI )and biofilm formation. Biofilm formation increased with duration of catheter in situ, but no significant correlation was found $(\mathrm{p}=0.095)$. This could be due to small number of specimens. $9 / 20(45 \%)$ urine samples, $12 / 20(60 \%)$ catheter samples were positive by culture and 14/20(70\%) catheters showed biofilm on SEM. 4/12(33.33\%) organisms isolated from catheter culture produced biofilm by TCP method. 9 isolates were recovered from 9 positive urine cultures. The microorganisms isolated were non Candida albicans (3/9), E. coli (2/9), C. albicans (2/9) and Acenitobacter (2/9). 14 isolates were recovered from 12 culturepositive catheters. The organisms isolated were E. coli (3/14), non-Candida albicans (3/14), C. albicans (2/14), C tropicalis (2/14), Acenitobacter (2/14), Klebsiella (1/14) and Enterococcus (1/14). Reduction in microbial diversity with antimicrobial use was noticed but the correlation was insignificant $(\mathrm{p}=0.317)$. The correlation between urine culture results as well as catheter culture results and biofilm formation by SEM were both significant $(\mathrm{p}=0.008 \& 0.000$ respectively). The correlation between urine culture and TCP assay was insignificant $(\mathrm{p}=0.237)$. Using SEM as the gold standard method for the detection of biofilm, the sensitivity, specificity, total accuracy, PPV \& NPV of urine culture and catheter culture were, $64.30 \%, 100 \%, 75 \%$, $100 \%, 54 \%$ \& $85.70 \%, 100 \%, 90 \%, 100 \%, 75 \%$ respectively.
\end{abstract}

Keywords: Urinary tract infection, biofilm formation, electron microscopy

\section{Introduction}

A biofilm is an aggregate of microorganisms embedded within a self-produced matrix of extracellular polymeric substance (EPS). Biofilm EPS is a polymeric conglomeration generally composed of extracellular DNA, proteins, and polysaccharides (Abd El-Baky, 2012).

Biofilm protects the bacterial community from environmental stresses, from host immune system, and from antibiotic attacks. Presence of biofilm is associated with increased bacterial resistance to antimicrobial therapy and resultant treatment failure which undermine patient quality of life and threatens the health of many people (Stickler, 2008).
Biofilm formation is recognized as causing or exacerbating numerous chronic infections including periodontitis, device-related infections, cystic fibrosis pneumonia, chronic urinary tract infections, recurrent tonsillitis, chronic rhinosinusitis and chronic wound infections (Peters et al, 2012). Biofilm formation on indwelling urinary catheters is a leading cause of urinary tract infections in hospitalized patients. Microbiological examination of urine from patients is often misleading, due to formation of these biofilms. Thus, characterizing these biofilms and identifying the microorganisms on surface of catheters were of major importance (Djeribi et al, 2012).

In this study, characterization of the biofilm communities, SEM and TCP were used to find 
out the incidence of biofilm formation in urinary catheterized patients and to detect a reliable diagnostic technique for the detection of biofilm formation.

\section{Patients, Materials and Methods}

Subjects studied were twenty in-patients with a mean age of $34.75 \pm 3.11$ years, median age was 28.50 years and mode was 1.50 years. Minimum age was 1.5 years and the maximum age was 85 years. Twelve of 20 patients were males $(60 \%)$ while 8 were females $(40 \%)$. Ten were from the Paediatric Surgical Ward at the New Children Hospital and ten from the Medical Ward at Internal Medicine Hospital, Cairo University, from February 2013 to August 2013. All were undergoing urethral catheterization. Urinary catheters durations in situ for adult patients ranged from 3-20 days with a mean of $11.6 \pm 5.3$ days, while for paediatric ones ranged from 3-6 days with a mean of $4.4 \pm 0.97$ days.

Full medical history was taken from each patient. Most of the patients were on antibiotics $(90 \%)$ and only a few $(10 \%)$ were not taking antibiotics at the time of data collection. Some adult patients had associated medical conditions (Diabetes mellitus, renal insufficiency, diarrhea and impaired immunity) that predispose to UTI and biofilm formation.

Twenty clinical samples (urine samples and catheter segments) were collected. Urine samples were aspirated from the catheters at same time of catheters removal, streaked onto blood and CLED agar and incubated at $37^{\circ} \mathrm{C}$ for 24 hours. The revealed colonies were examined morphologically, microscopically and biochemically. Latex or silicone coated Foley catheters were freshly removed from patients under aseptic conditions. Details of the length of time each catheter was indwelling and any current antibiotic therapy was also registered.

Characterization of Biofilm Communities: To characterize bacterial colonizing the lumen of the catheters, sections ( $1 \mathrm{~cm}$ long) were cut from the region of the catheter within retention balloon using sterile scissors. Each section was immediate-ly rinsed gently in $10 \mathrm{ml}$ of buffer (Hanks balanced salt solution, $\mathrm{pH}$ 7.4) (SigmaAldrich, USA) and put in $10 \mathrm{ml}$ of nutrient broth (Oxoid). Each tube of nutrient broth underwent sonication for 1 minute, followed by vortex mixing for another minute to disrupt the luminal biofilm.

Samples of the broth suspensions were then plated out on blood \& CLED agar and incubated for 24 hours at $37^{\circ} \mathrm{C}$. Microorganisms were identified by routine microbiological techniques (Roland and James, 2011). Candida species were identified by CHROM agar candida media to differentiate species based on color development on agar (Singh et al, 2013).

Scanning Electron Microscopy (SEM): To detect biofilm by SEM, sections of catheters $(1 \mathrm{~cm}$ in length) were taken from the part adjacent to retention balloon on the side away from the catheter tip. Sections were first immediately fixed for 2 hours in equal volumes of glutaraldehyde $4 \%$ and caccodylate $0.2 \mathrm{M}$, washed in equal volumes of sacchrose $0.4 \mathrm{M} \&$ caccodylate $02 \mathrm{M}$ for 2 hours and then post fixed in osmium tetroxide $2 \%$ and caccodylate $0.3 \mathrm{M}$ for an hour. The samples were then washed with distilled water and finally dehydrated in ascending grades of ascending ethanol for 5 minutes each then absolute ethanol for 10 minutes for 3 times. Specimens were examined with Philips XL30 (Eindhoven, the Netherlands) SEM operated at $10-30 \mathrm{KV}$, EM unit of Theodor Bilharz Research Institute.

Detection of Biofilm Formation by TCP: Semi-quantitative determination of biofilm formation was based on the techniques (Stepanovic et al, 2007). Tested strains were frozen and maintained at $-80^{\circ} \mathrm{C}$, then subcultured on CLED agar plates overnight aerobically at $37^{\circ} \mathrm{C}$ prior to each assay. Four identical colonies from each plate were suspended in $5 \mathrm{ml}$ TSB and incubated without shaking for 24 hours then vortexed. The wells of sterile, polystyrene flat-bottomed 
96-well microtiter tissue culture plate was filled with $180 \mu \mathrm{L}$ of TSB. $20 \mu \mathrm{L}$ of bacterial suspension were added to each well. Six negative control wells containing $200 \mu \mathrm{L}$ of TSB were included. The inoculated plate was covered and incubated aerobically for 24 hours at $37^{\circ} \mathrm{C}$ under static conditions. The turbidities in the wells were measured using a microtiter plate reader (STAT FAX 2100 ) at $600 \mathrm{~nm}$ wavelength to measure bacterial growth in the wells. Each well was washed three times with $300 \mu \mathrm{L}$ of PBS (pH 7.2). The plate was drained in an inverted position. Adherent biofilms in wells were fixed by the addition of $50 \mu \mathrm{L} 95 \%$ ethanol to each well, incubated for 30 minutes then emptied by simple flicking and air dried. 50 $\mu \mathrm{L} 0.06 \%(\mathrm{w} / \mathrm{v})$ solution of crystal violet dissolved in deionized water $\left(\mathrm{dH}_{2} \mathrm{O}\right)$ were added to each well and allowed to stain for 60 minutes. Excess stain was removed by adding $200 \mu \mathrm{L}$ of $\mathrm{dH}_{2} \mathrm{O}$ to each well and decanted. The process was repeated four times. The wash solution in t final washing step was clear. After drying, the optical density (OD) of each well stained with crystal violet was measured at $600 \mathrm{~nm}$ using the same microtiter plate reader.

Interpretation of the results: Each assay was performed in triplicate. As a negative control, uninoculated medium was used to determine background OD. The average OD values were calculated for all tested strains and negative controls, the cut-off value (ODc) was established. It is defined as a three standard deviations (SD) above the mean OD of the negative control: ODc $=$ average $\mathrm{OD}$ of negative control $+(3 \times \mathrm{SD}$ of negative control). ODc value was calculated for each microtiter plate separately. When a negative value was obtained and presented as zero, while any positive value indicated biofilm production.

For interpretation of the results, strains were divided into the following categories: 1$\mathrm{OD} \leqslant \mathrm{ODc}=$ Non biofilm producer, 2$\mathrm{ODc}<\mathrm{OD} \leqslant 2 \times \mathrm{ODc}=$ Weak biofilm producer, $3-2 \times \mathrm{ODc}<\mathrm{OD} \leqslant 4 \times \mathrm{ODc}=$ Moderate biofilm producer, and $4-4 \times \mathrm{ODc}<\mathrm{OD}=$ Strong biofilm producer.

\section{Results}

Of 10 adult patients studied, 5 were males and 5 females, with ages ranged from 61-75 years. Of 10 paediatric patients, 7 were males \& 3 were females, age ranged from 14 years. Three adult patients had intracranial haemorrhage (ICH), 3 had hepatic encephalopathy (HE) and 4 had meningitis, Diabetic keto acidosis, renal failure and cardiomyopathy. Ten pediatric patients were postoperative and 8 were diagnosed as renal calculi, one had brain tumor and one had congenital bladder anomaly. Some adults had associated medical conditions (Diabetes mellitus, renal insufficiency, diarrhea and impaired immunity) that predispose to UTI and biofilm formation, 3/10 adults had Diabetes mellitus and 1/10 had renal insufficiency. None of pediatric patients had associated medical condition. Patients $(90 \%)$ were on antibiotics and $10 \%$ were not at data collection time. $4 / 20(20 \%)$ of patients took antibiotics for two weeks, 4/20 (20\%) took for 3 days, 3/20 (15\%) took for 5 days, 3/15 (15\%) took for 2 days, $2 / 20(10 \%)$ took for 7 days, $1 / 20(5 \%)$ took for 6 days and 1/20 $(5 \%)$ took for 4 days. Two patients were not on antibiotic therapy at catheters removal. Durations of urinary catheters in situ for the adults ranged from 3-20 days (11.6 \pm 5.3 ), while ones for pediatric patients ranged from 3-6 days (4.4 \pm 0.97$)$.

Urine culture: $E$. coli was isolated from 2/20 (10\%) samples, Acinetobacter from 2/ 20 (10\%), C. albicans from $2 / 20(10 \%)$, and Non C. albicans from 3/20 (15\%) samples, 11/20 (55\%) without growth (Tab. 1).

Catheter culture: $10 / 20 \quad(50 \%)$ cultures showed pure cultures of E. coli, Klebsiella, Acinetobacter, C. albicans, C. tropicalis and Non $C$. albicans. 2/20 (10\%) cultures showed mixed cultures and $8 / 20(40 \%)$ samples without growth. Escherichia coli and Non Candida albicans were commonest isolated from $6 / 20(30 \%)$ of the catheters $(3[15 \%]$ 
for each organism). Bacteriuria was less common than biofilm colonization and some like C. tropicalis, Klebsiella and Enterococcus were not isolated from samples (Tab. 2).

SEM analysis of biofilms formed on inner surfaces of catheter samples showed a dense network of cellular multilayers surrounded by protective matrix with intercellular pores and interconnected channels as primitive circulatory system which allow the flow of water and ions, the transport of nutrients and the efflux of waste products. $14 / 20(70 \%)$ catheter samples showed bio-film formation. Catheters were indwelling in situ for durations ranged from 3-20 days (mean $9 \pm 5.42$ days), and the patients from which samples were taken, were on antibiotics for durations which ranged from 0 -14 days $(6.14 \pm 4.68)$. $6 / 20(30 \%)$ were without biofilm (Tab. 3). The in situ duration ranged from 3-14 days (5.67 \pm 4.22$)$, and patients were on antibiotics for durations from 0-14 days (4.5 \pm 4.92$)$.

SEM analysis of the biofilms formed on the inner surfaces of the catheter samples revealed a dense network of cellular multilayers, formed either from a single cell (Fig 1) or from different species (Fig 2,3), surrounded and enveloped by a protective matrix. SEM images indicate that the identified isolated species readily developed compatible and strong associations with other species and formed agglomerates on the inner catheter surfaces. Biofilms were characterized by a multitude of intercellular pores and interconnected channels did allow the exchange of information and the flow of water and ions, but transport of nutrients and the efflux of waste products (fig 2, 4).

Correlation between duration of catheter in situ and biofilm formation: Catheters showed biofilm (7/14) were indwelling for 3-7 days and most of biofilm free catheters $(5 / 6)$ were also in situ for same duration, without significant ( $\mathrm{p}=0.095)$ (Tab. 4).

Correlation between associated conditions and biofilm formation by SEM: Three patients with DM 3/20 (15\%) had biofilm and $1 / 20(5 \%)$ with renal insufficiency, had no biofilm. For 16/20 (80\%) without predisposing factors, $5 / 16(31.25 \%)$ had no biofilm and 11/16 (68.75\%) had biofilm. The correlation was insignificant $(\mathrm{p}=0.163)$.

Correlation between antibiotic treatment and biofilm formation: 18/20 (90\%) patients took antibiotics at catheter removal, 13/18 had positive catheter cultures. $8 / 18$ had bacteriuria $\left(>10^{3} \mathrm{cfu} / \mathrm{ml}\right) \& 5 / 18$ without bacteriuria. Most cultures had single bacterial isolate; mixed ones were in 2 samples.

Correlation between duration of antibiotic intake and biofilm formation: 14/20(70\%) of patients had positive biofilm by SEM, 8 of them took antibiotics for durations that ranged from 0-5 days. One patient did not take antibiotics. For $6 / 20(30 \%)$ patients who had no biofilm, 5 took antibiotics for durations of 0-5 days and one patient did not take antibiotics, without significant (Tab. 5).

Correlation between urine culture and biofilm formation by SEM: In 14/20 (70\%) catheters showed biofilm. Organisms were isolated from $9 / 20(45 \%)$ urine samples and $11 / 20(55 \%)$ of urine had no growth on culture, with significant $(\mathrm{p}=0.008)$.

Correlation between catheter culture and biofilm by SEM: Organisms isolated from culture of $12 / 20(60 \%)$ catheters were Klebsiella, E.coli, C. tropicalis, C. albicans, Non C. albicans, Enterococcus and Acinetobacter, $2 / 20(10 \%)$ samples showed no culture growth but showed biofilm by SEM with strongly significant ( $p=0.000)$ (Fig. 11).

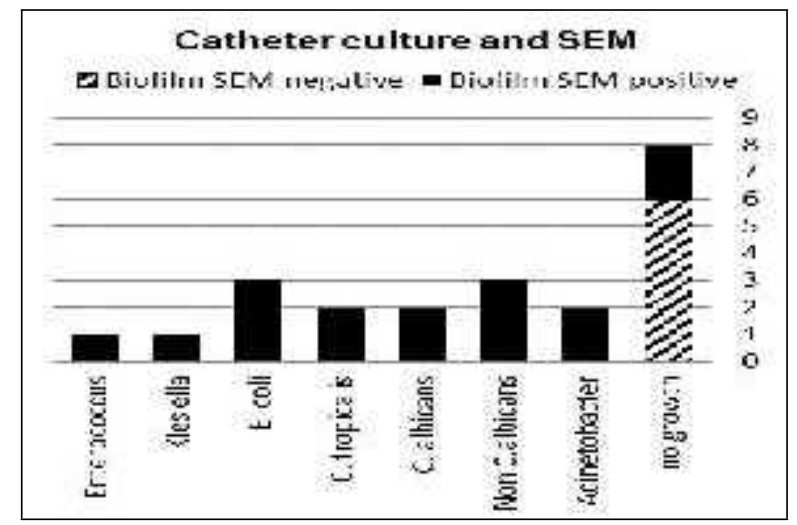

Fig. 9: Correlation between catheter culture and biofilm formation by SEM (20 cases). 
TCP assay: Using the semi-quantitative 96well plate assay, microorganisms isolat-ed from 12/20 (60\%) catheter samples were tested in vitro for their ability to produce biofilm by TCP assay. One isolate non $C$. albicans was strong biofilm producer, another non $C$. albicans and $C$. tropicalis were moderate producers. Enterococcus was a weak biofilm produ-cer. 4/12 (33.33\%) isolates from urinary catheters luminal surfaces were biofilm producers, 8/12 (66.66\%) samples were E. coli, Klebsiella, Acinetobacter, C. albicans were non biofilm producers (Tab. 6). Organisms from urine culture were 2 E. coli, 2 C. albicans, 3 non $C$. albicans and 2 Acinetobacter. Only 2/9 isolated organisms were able to produce biofilm by TCP and both of them were non $C$. albicans. (1/3) of non C. albicans was a strong biofilm producer, $(1 / 3)$ was a moderate biofilm producer \& (1/3) was a non-biofilm producer, without significant $(\mathrm{p}=0.237)$.

Correlation between catheter culture, SEM \& TCP assay: 14/20 (70\%) were biofilm producers. $4 / 14$ (28.57\%) samples, biofilm formation was detected by SEM and TCP assay. 10/14 (71.43\%) samples, biofilm formation detected by SEM but isolates from those biofilms did not produce biofilm by TCP assay. $6 / 20(30 \%)$ were non biofilm producer by SEM and TCP assay (Tab. 7).

Of $8 / 20(40 \%)$ the catheter samples showed positive biofilm by both microbiological culture and SEM, but were not the biofilm producers by the TCP assay (Tab. 8). 6/20
$(30 \%)$ of samples showed no organisms neither by routine culture methods and accordingly by TCP assay, nor SEM. 6/20 $(30 \%)$ of samples, the isolates from catheter culture match with those by SEM. Another $6 / 20(30 \%)$ catheter samples showed no growth by culture and none by SEM. In $8 / 20$ $(40 \%)$ of catheter samples, isolates from culture did not match with microorganism type by SEM. SEM data evaluated urine culture, catheter culture and TCP methods. Urine culture had a sensitivity of $64.30 \%$, specificity of $100 \%$, total accuracy of $75 \%$, PPV \& NPV of $100 \%$ \& $54.5 \%$ respectively. Catheter culture had a sensitivity of $85.70 \%$, specificity of $100 \%$ and total accuracy of $90 \%$. PPV and NPV were $100 \%$ and $75 \%$ respectively.

The sensitivity of TCP assay against SEM could not be statistically calculated because there were no organisms that were biofilm producers by TCP method and non-biofilm producers by SEM, i.e. no negative results.

The details were given in tables $(1,2,3,4$, $5,6,7 \& 8)$ and figures $(1,2,3,4,5,6,7,8$ \& 9).

Table 1: Organisms isolated from 20 urine samples

\begin{tabular}{|c|c|c|}
\hline Organisms & Samples No. & $\begin{array}{c}\text { Percentage } \\
(\%)\end{array}$ \\
\hline E. coli & 2 & 10 \\
\hline Acinetobacter & 2 & 10 \\
\hline C. albicans & 2 & 10 \\
\hline Non C. albicans & 3 & 15 \\
\hline No growth & 11 & 55 \\
\hline & Total $=20$ & Total $=100$ \\
\hline
\end{tabular}

Table 2: Culture of 20 catheter samples.

\begin{tabular}{|c|c|}
\hline No. of samples & Culture \\
\hline 2 & Pure E. coli \\
\hline 1 & Pure Klebsiella \\
\hline 2 & Pure Acinetobacter \\
\hline 2 & Pure C. albicans \\
\hline 1 & Pure C. tropicalis \\
\hline 2 & Pure Non C. albicans \\
\hline 1 & Mixed culture E. coli + C. tropicalis) \\
\hline 1 & Mixed culture (Non C. albicans + Enterococcus) \\
\hline 8 & Nowth \\
\hline Total=20 & \\
\hline
\end{tabular}


Table 3: SEM for 20 samples

\begin{tabular}{|c|c|c|c|}
\hline Cases & \multicolumn{2}{|c|}{ Biofilm } & \multirow{2}{*}{ Total } \\
\hline & Positive & Negative & \\
\hline Paediatric & 6 & 4 & 10 \\
\hline Adult & 8 & 2 & 10 \\
\hline Total & 14 & 6 & 20 \\
\hline
\end{tabular}

Table 4: Correlation between duration of catheter in situ and biofilm formation in e 20 samples

\begin{tabular}{|c|c|c|}
\hline \multirow{2}{*}{ Days of catheter in situ } & \multicolumn{2}{|c|}{ Biofilm } \\
\cline { 2 - 3 } & Positive & Negative \\
\hline $3-7$ & $7(35 \%)$ & $5(25 \%)$ \\
\hline $8-12$ & $3(15 \%)$ & $0(0 \%)$ \\
\hline $13-17$ & $3(15 \%)$ & $1(5 \%)$ \\
\hline$>18$ & $1(5 \%)$ & $0(0 \%)$ \\
\hline & $14(70 \%)$ & $6(30 \%)$ \\
\hline
\end{tabular}

Table 5: Correlation between antibiotic intake duration and biofilm formation ( 20 cases)

\begin{tabular}{|c|c|c|}
\hline \multirow{2}{*}{ Duration of antibiotic intake/Days } & \multicolumn{2}{|c|}{ Biofilm } \\
\cline { 2 - 3 } & Positive & Negative \\
\hline $0-5$ & 8 & 5 \\
\hline $6-10$ & 3 & 0 \\
\hline $11-15$ & 3 & 1 \\
\hline & Total=14 & Total=6 \\
\hline
\end{tabular}

Table 6: Organisms isolated from 12 catheters biofilm producers by TCP assay.

\begin{tabular}{|c|c|c|c|}
\hline Microorganisms & $\begin{array}{c}\text { Organisms isolated } \\
\text { from catheter culture }\end{array}$ & \multicolumn{2}{|c|}{ Biofilm producing organisms } \\
\cline { 3 - 4 } & 3 & No. & $\%^{*}$ \\
\hline E. coli & 1 & 0 & $0 \%$ \\
\hline Enterococcus & 1 & 0 & $100 \%$ \\
\hline Klebsiella & 2 & 0 & $0 \%$ \\
\hline Acinetobacter & 2 & 0 & $0 \%$ \\
\hline C. albicans & 1 & 1 & $0 \%$ \\
\hline C. tropicalis & 2 & 2 & $100 \%$ \\
\hline Non C. albicans & 12 & 4 & $100 \%$ \\
\hline Total & 12 & \\
\hline
\end{tabular}

*Percent was correlated to number of each isolate.

Table 7: Comparison between results of biofilm detection by SEM and TCP assay ( $\mathrm{n}=20)$.

\begin{tabular}{|c|c|c|}
\hline Total No. of isolates & SEM & TCP assay \\
\hline 4 & + & + \\
\hline 6 & - & - \\
\hline 10 & + & - \\
\hline Total $=20$ & 14 & 4 \\
\hline
\end{tabular}

Table 8: Correlation between catheter culture, SEM and TCP assay.

\begin{tabular}{|c|c|c|c|c|}
\hline & Catheter culture & \multicolumn{2}{|r|}{ SEM } & Biofilm production by TCP assay \\
\hline & & Biofilm & Microorganism shape & \\
\hline 1 & C. tropicalis & Positive & Non & - \\
\hline 2 & Klebsiella & Positive & Non & No biofilm producer \\
\hline 3 & Acinetobacter & Positive & Coccobacilli & No biofilm producer \\
\hline 4 & Non C. albicans/ Enterococcus & Positive & Non & -/Weak biofilm producer \\
\hline 5 & C. albicans & Positive & Non & No biofilm producer \\
\hline 6 & No growth & Negative & Non & - \\
\hline 7 & C. albicans & Positive & Hyphae & No biofilm producer \\
\hline 8 & No growth & Positive & Cocci & - \\
\hline 9 & Non C. albicans & Positive & Non & Moderate biofilm producer \\
\hline 10 & No growth & Negative & Non & - \\
\hline 11 & E. coli/C.tropicalis & Positive & Hyphae & No biofilm producer/moderate biofilm producer \\
\hline 12 & Non C. albicans & Positive & Non & Strong biofilm producer \\
\hline 13 & E. coli & Positive & Cocci and Bacilli & No biofilm producer \\
\hline 14 & E. coli & Positive & Cocci and Bacilli & No biofilm producer \\
\hline 15 & No growth & Negative & Non & - \\
\hline 16 & No growth & Positive & Non & - \\
\hline 17 & No growth & Negative & Non & - \\
\hline 18 & No growth & Negative & Non & - \\
\hline 19 & Acinetobacter & Positive & Coccobacilli & No biofilm producer \\
\hline 20 & No growth & Negative & Non & - \\
\hline
\end{tabular}




\section{Discussion}

Biofilms have effective defense mechanisms against the host immune system, against antimicrobial agents, and difficult to eradicate (Kaya et al, 2013). Biofilm organisms may elicit disease processes by detachment of individual cells or aggregates of cells from the device surface or by production of endotoxins or other pyrogenic substances. They provided a niche for development of antimicrobial-resistant organisms (Weinstein and Donlan, 2011). Due to the large number of infections caused by biofilm producing bacteria, a reliable method for diagnosis was necessary (Oliveira and Cunha, 2010).

Most patients with renal conditions had biofilm on luminal surface of their catheters. Correlation was not significant $(\mathrm{p}=0.336)$. But, the isolates from urine of patients with renal calculi were $100 \%$ biofilm producers (Maheswari et al., 2013).

In this study, catheters placed in situ 6 days or more all revealed biofilm formation, but there was no statistical significance between increased duration of catheters in situ and biofilm formation $(p=0.095)$. This could be due to small samples. Moreover, duration of catheter in situ is most important risk factor for development of bacteriurea and risk of catheter-associated urinary tract infection increases by an estimated $5 \%$ to $10 \%$ each day as long as the catheter remains in place, adding that patients with long-term catheters are almost assured of developing CAUTI (Chenoweth and Saints, 2013).

Biofilms were formed in patients on antibiotic therapy, but with low diversity maximum of two of the microbial species. Low diversity was due to antibiotics killing some species, short catheter indwelling period ( $\leq 20$ days) and microbial competition for resources. Xu et al. (2012) found that antibiotics reduced microbial species diversity preventing their colonization in shortterm catheterized patients, without relation between antibiotic duration and biofilm formation $(p=0.317)$. No correlation was found between urine culture results and any associated conditions (DM, renal insufficiency, diarrhea and impaired immunity) that could predispose to catheter-associated urinary tract infection (CAUTI) and hence biofilm formation $(p=0.836)$. This study showed increased catheter colonization rate with $\mathrm{DM}$, not significant $(\mathrm{p}=0.163)$ due to small sample size. Others (Akay et al, 2007; Niveditha et al, 2012; Lee et al, 2013) found DM significantly associated with UTI in catheterized patients and biofilm formation. More microorganisms were cultured. Catheters $12 / 20(60 \%)$ than in urine samples $9 / 20$ (45\%). This agreed with $\mathrm{Xu}$ et al. (2012) reported culture of catheter tips in more frequent positive 15/16 compared to urine culture $8 / 16$, commonest were E. coli $3 / 20$ and non-Candida albicans 3/20. Niveditha et al. (2012) found E.coli frequently isolated from 35/50 (70\%). P. mirabilis, P. stuartii, Morganella morganii and $K$. pneumoniae produce urease and form crystalline biofilms on catheters. Urease production destroyed urea and releases ammonia raised urine $\mathrm{pH}$ resulted in calcium \& magnesium phosphate crystal formation in biofilm matrix (Amalaradjou and Venkitanarayanan, 2013). SEM examined urinary catheters for crystalline biofilms (Stickler and Morgan, 2006). They isolated Klebsiella from catheter culture, $4 / 20$ of biofilms SEM were crystalline in nature. In urinary catheters of hospitalized patients, SEM showed that many catheters removed after a week were colonized by biofilms on luminal surfaces, different species readily integrated and develop close associations in biofilms (Djeribi et al, 2012). Some urine samples were free of microorganisms, but catheters showed biofilm on SEM. Urine culture matched with SEM results of catheter samples, with significant $(p=0.008)$. No positive urine cultures have biofilm by SEM. Positive culture catheter samples revealed biofilm by SEM with significant $(p=0.000)$ but vise was not versa. Semi-quantitative microtitre TCP assay is a reliable and reproducible method for assessing biofilm formation in vitro (Mathur 
et al, 2006). Standard trypticase soy broth medium (without sugar) was used and 4/12 were biofilm producers. Los et al. (2010) found 52/146 using TSB. Supplementation of TSB with glucose enhances the biofilm producing capacity of bacteria (Revdiwala et al, 2012).This was done on Enterococcus faecium strains (Diani et al, 2014) and on staphylococcal isolates by Agarwal and Jain (2013). Terki et al. (2013) showed that 8/44 isolates were biofilm producers with standard TSB and 15/44 after glucose supplementation. Biofilm by TCP method was not dependent on composition of medium, but on constitutional and environmental factors and host proteins that drastically changed results (Agarwal and Jain, 2013). Biofilm producers by TCP were two non C. albicans (one strong and one moderate) isolated from urine and catheter cultures, C. tropicalis (moderate) and enterococcus (weak). Non $C$. albicans had greater biofilm-forming ability than $C$. albicans. This agreed with Kaur et al. (2014). Some microorganisms isolated from urine culture produced biofilm by TCP assay but without significant. All biofilm producing organisms by TCP assay were biofilm producers by SEM, but could not be calculated as none was biofilm producers by TCP nor by SEM. SEM proved to be good to detect biofilms on catheters, elucidating their structure for understanding physiology and ecology of their systems (El Abed et al, 2012, Singhai et al, 2012; Dawei et al, 2012).

\section{Conclusion}

Nosocomial infection is associated with urinary catheters due to bacterial biofilms on their surface. The use of systemic antibiotics did not alter biofilm formation. Urine culture, often gave misleading biofilm. More microorganisms were isolated from catheter culture than from urine culture. TCP assay is a screening test for biofilm detection. SEM is valuable for characterization of biofilms on catheters and identification of infectious agents responsible for UTI and biofilm formation, Microbiological analysis of the inner catheter surface combined with microbiological examination of urine, allow for identification and complete characterrization of the bacterial species forming the biofilm.

\section{References}

Abd El-Baky, RM, 2012: Application of scanning electron microscopy for the morphological study of biofilm in medical devices. In: Kazmiruk V: Scanning Electron Microscopy.

Agarwal, A, Jain, A, 2013: Glucose \& sodium chloride induced biofilm production \& operon in clinical isolates of staphylococci. Indian J. Med. Res. 262-266.

Akay, AF, Aflay, U, Gedik, A, Sahin, H, Birkan, MK, 2007: Risk factors for lower urinary tract infection and bacterial stent colonization in patients with a double $\mathrm{J}$ ureteral stent. Int. Urol. Nephrol. 39, 1:95-8.

Amalaradjou, MA, Venkitanarayan, K, 2013: Role of bacterial biofilms in catheter-associated urinary tract infections (CAUTI) and strategies for their control. In: Nelius $T$ (Eds): Recent advances in the field of urinary tract infections.

Chenoweth, C, Saint, S, 2013: Preventing catheter-associated urinary tract infections in the ICU. Crit. Care Clin. 29, 1:34-9.

Dawei, G, Liping, W, Chengping, L, 2012: In vitro biofilm forming potential of Streptococcus suis isolated from human and swine in China. Brazilian J. Microbiol. 43, 3:993-1004.

Diani, M, Esiyok, OG, Ariafar, MN, Yukse, F N, Altuntas, EG, et al, 2014: Interactions between esp, fsr, gelE genes and biofilm formation and pfge analysis of clinical Enterococcus faecium strains. Afric. J. Microbiol. Res. 8, 2: 12937.

Djeribi, R, Bouchloukh, W, Jouenne, T, Menaa, B, 2012: Characterization of bacterial biofilms formed on urinary catheters. American J. Infect. Cont. 40:854-9.

El Abed, S, Ibnsouda, SK, Latrache, H, Hamadi, F, 2012: Scanning electron microscopy (SEM) and environmental SEM: Suitable tools for study of adhesion stage and biofilm formation. In: Kazmiruk V: Scanning Electron Microscopy.

Kaur, R, Goya, R, Dhakad, MS, Bhalla, P, Kumar, R, 2014: Epidemiology and virulence determinants including biofilm profile of candida infections in an ICU in a tertiary hospital in India. J. Mycol. 3:1-8.

Kaya, E, Dag, I, Incesulu, A, Gurbuz, MK, Acar, M, Birdane, M, 2013: .Investigation of presence of biofilms in chronic suppurative otitis 
media, nonsuppurative otitis media, and chronic otitis media with cholesteatoma by scanning electron microscopy. Sci. Wld. J. 638715:1-6.

Lee, JH, Kim, SW, Yoon, B, Ha, US, Sohn, DW, et al, 2013: Factors that affect nosocomial catheter-associated urinary tract infection in intensive care units: 2-Year experience at a single center. Korean J. Urol. 54, 1:59-65.

Los, R, Sawicki, R, Juda, M, Stankevic, M, Rybojad. $P$, et al, 2010: A comparative analysis of phenotypic and genotypic methods for the determination of the biofilm-forming abilities of Staphylococcus epidermis. Federation of European Microbiological Societies 310:97-103.

Maheswari, UB, Palvai, S, Anuradha, PR, Kammili N, 2013: Hemagglutination and biofilm formation as virulence markers of uropathogenic Escherichia coli in acute urinary tract infections and urolithiasis. Indian J. Urol. 29, 4: 277-81.

Mathur, T, Singhal, S, Khan, S, Upadhyay, D J, Rattan, A, 2006: Detection of biofilm formation among the clinical isolates of Staphylococci: An evaluation of three different methods. Indian J. Med. Microbiol. 24, 1:25-29.

Niveditha, S, Pramodhini, S, Umadevi, S, Kumar, S, Stephen. S, 2012: The isolation and the biofilm formation of uropathogens in the patients with catheter associated urinary tract infections. J. Clin. Diag. Res. 6, 9:1478-82.

Oliveira, A, Cunha, MRS, 2010: Comparison of methods for the detection of biofilm production in coagulase-negative staphylococci. BMC Res. Notes 3:260-8.

Peters, BM, Jabra-Rizk, MA, O'May, GA, Shirtliff, ME, 2012: Polymicrobial Interactions: Impact on pathogenesis and human disease. Clin. Microbiol. Rev. 25, 1:193-213.

Revdiwala, S, Bhaumesh, MR, Summaiya, M, 2012: Characterization of bacterial etiologic agents of biofilm formation in medical devices in critical care setup. Crit. Care Res. 945805:1-6.

Ronald, MA, James, WS, 2011: Reagents, stains, and media: Bacteriology. In Manual of Clinical Microbiology, $10^{\text {th }}$ ed.

Singh, G, Raksha, Urhekar, AD, 2013: Candidal infection: epidemiology, pathogenesis and recent advances for diagnosis, a peer reviewed. Int. J. 1, 1:1-8.

Singhai, M, Malik, A, Shahid, M, Malik, MA, Goyal, R, 2012: A Study on device-related infections with special reference to biofilm production and antibiotic resistance. J. Glob. Infect. Dis. 4, 4:193-8.

Stepanović, S, Vucović, D, Hola, V, Di Bonaventura, G, et al, 2007: Quantification of biofilm in microtiter plates: overview of testing conditions and practical recommendations for assessment of biofilm production by staphylococci. Acta Pathol. Microbiol. Immunol. Scand. 115, 8:891-9.

Stickler, DJ, Morgan, SD, 2006: Modulation of crystalline Proteus mirabilis biofilm development on urinary catheters. J. Med. Microbiol. 55: 489-94.

Stickler, DJ, 2008: Bacterial biofilms in patients with indwelling urinary catheters. Nat. Clin. Pract. Urol. 5, 11:45-9.

Terki, IK, Hassaine, H, Oufrid, S, Bellifa, S, Mhamedi. I, et al, 2013: Detection of ica $\mathrm{A}$ and $i c a D$ genes and biofilm formation in Staphylococcus spp. isolated from urinary catheters at the University Hospital of Tlemcen (Algeria). Afri. J. Microbiol. Res. 7, 47:5350-7.

Weinstein, RA, Donlan. RM, 2011: Biofilm elimination on intravascular catheters: important considerations for the infectious disease practitioner. Clin. Infect. Dis. 52, 8:1038-45.

Xu, Y, Moser, C, Abu Al-Soud, W, Sørensen S, et al, 2012: Culture-dependent and independent investigations of microbial diversity on urinary catheters. J. Clin. Microbiol. 50, 12:3901-8.

\section{Explanations of figures}

Fig. 1: SEM showing a dense mass of bacterial biofilm with large cocci, Duration of catheterization 4 days (2000x).

Fig. 2: SEM of biofilm with scattered microorganisms, intercellular pores and interconnected channels, resembling primitive circulatory system which allow flow of water and ions, transport of nutrients and efflux of waste products. Duration of catheterization was 5 days.

Fig. 3: SEM of a non-crystalline mature biofilm caused by candida and chains of cocci mostly enterococci (1200x). Catheter removed after 10 days.

Fig. 4: SEM of a dense mass of biofilm containing bacteria, intercellular pores and interconnected channels at higher magnification (1000x).

Fig. 5: SEM showing candida at higher magnification. No crystalline material was deposited in bacterial biofilm. Duration of catheterization was 5 days.

Fig. 6: SEM of a high dense crystalline biofilm. Catheter removed after 5 days. Bacilli and cocci colonizing a microcrystalline foundation layer on catheter inner surface $(600 x)$.

Fig. 7: SEM of same crystalline biofilm at higher magnification (1200x) caused by urease positive organisms. Catheter removed after 5 days. Fig. 8: SEM of a crystalline biofilm caused by urease positive organisms (1200x). Microcrystalline aggre-gates associated with scattered cocci. Catheter removed after 5 days. 

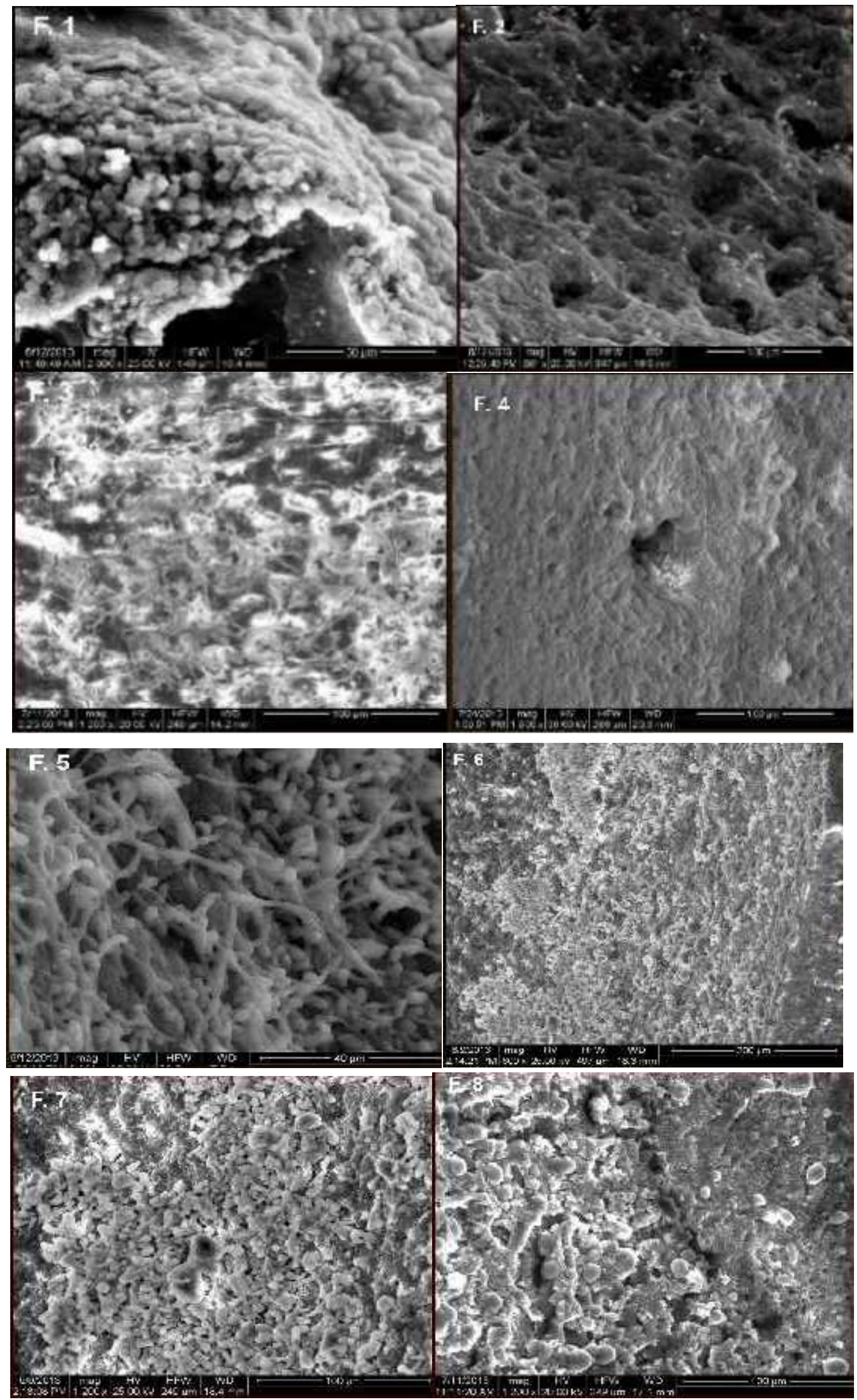